\title{
SÍNDROMES DE DISPERSÃO DE ESPÉCIES ARBÓREAS DE FLORESTAS OMBRÓFILAS SUBMONTANAS DO ESTADO DO RIO DE JANEIRO ${ }^{1}$
}

\author{
Fabrício Alvim Carvalho²
}

\begin{abstract}
RESUMO - O objetivo deste trabalho foi verificar as diferenças nas proporções das síndromes de dispersão de propágulos de espécies arbóreas entre florestas ombrófilas submontanas secundárias e preservadas no Estado do Rio de Janeiro. Foram utilizadas listas de espécies de sete florestas secundárias e cinco preservadas. Partiuse da hipótese de que as florestas secundárias apresentariam menor riqueza e densidade de espécies arbóreas com síndrome de dispersão biótica (zoocoria). As médias das proporções de árvores com síndrome de dispersão biótica entre florestas secundárias e preservadas foram comparadas pelo teste U. Apesar de preponderarem em ambas, florestas secundárias e preservadas diferiram significativamente em riqueza e densidade de espécies com dispersão biótica $(\mathrm{P}<0,01)$. Essas proporções foram menores nas florestas secundárias, corroborando essa hipótese. As florestas secundárias também apresentaram menor densidade de espécies zoocóricas pertencentes a famílias dispersadas por grandes vertebrados frugívoros (Lauraceae, Myrtaceae e Sapotaceae). Futuras práticas de manejo e conservação nessas florestas secundárias devem incorporar as interações plantas-dispersores, devido aos riscos nos processos de regeneração sem a presença da fauna dispersora adequada.
\end{abstract}

Palavras-chave: Floresta Atlântica, Dispersão de sementes e Zoocoria.

\section{DISPERSAL SYNDROMES OF WOODY SPECIES IN THE SUBMOUNTAINE OMBROPHILOUS FORESTS OF THE RIO DE JANEIRO STATE, BRAZIL}

\begin{abstract}
This study aimed to evaluate the differences between propagule dispersal syndromes of woody species in secondary and mature submountaine ombrophilous forests in the State of Rio de Janeiro, Brazil. The hypothesis is that secondary forests will show lower richness and density of woody species with biotic seed dispersal modes (zoocory). Lists of seven secondary and five mature forests were used. The averages of the proportions of biotic modes between secondary and mature forests were compared through U test. Secondary and mature forests differed statistically in richness and density of biotic species $(P<0.01)$. These proportions were lower in the secondary forests, according to the hypothesis. Secondary forests also showed a decrease in the density of zoocoric species dispersed by large vertebrates, belonging to Lauraceae, Myrtaceae e Sapotaceae. Further management and conservation practices in these secondary forests must need incorporate the plantdispersers interactions, due to risks in the forest regeneration process without the adequate dispersal fauna.
\end{abstract}

Keywords: Atlantic Forest, Seed dispersal and Zoocory.

\section{INTRODUÇÃO}

A dispersão de propágulos é um dos processos mais importantes na manutenção florestal, influenciando diretamente a estrutura e dinâmica das comunidades vegetais (HOWE SMALLWOOD, 1982; WUNDERLE-Jr., 1997). A dispersão de propágulos ocorre de várias maneiras, e, de acordo com van der Pijl (1982), as três principais estratégias são: anemocoria, quando o propágulo é dotado de estruturas que facilitam o transporte pelo vento; autocoria, quando apresenta mecanismos próprios para o lançamento dos frutos ou sementes; e zoocoria, quando a dispersão é realizada por animais.

A importância dessas diferentes estratégias de dispersão pode variar de acordo com o tipo de vegetação e com o seu estádio sucessional (VIEIRA e SCARIOT, 2006; CHAZDON et al., 2007). Em florestas tropicais,

${ }^{1}$ Recebido em 24.02.2008 e aceito para publicação em 25.08.2010.

${ }^{2}$ Departamento de Botânica, Universidade Federal de Juiz de Fora, UFJF, Brasil.. E-mail: <fabricio.alvim@gmail.com>. 
a proporção de espécies arbóreas zoocóricas decresce gradativamente das regiões úmidas para as secas, e nas florestas mais úmidas (ombrófilas) a proporção de espécies zoocóricas normalmente ultrapassa os 80\% (GENTRY, 1982). Com relação ao estágio sucessional, a proporção de espécies zoocóricas costuma ser menor em florestas mais perturbadas e em estádios iniciais de sucessão (secundárias), em comparação com florestas preservadas em estádios mais avançados (maduras) (BROWN e LUGO, 1990; CHAZDON et al., 2007).

Como as florestas tropicais apresentam elevada proporção de espécies zoocóricas, a fauna dispersora é determinante para a manutenção das comunidades arbóreas em longo prazo (HOWE e SMALLWOOD, 1982; WUNDERLE-Jr., 1997). Diversos estudos vêm indicando que a fauna dispersora é suscetível a perturbações antrópicas, e isso implica problemas na regeneração e funcionamento das comunidades florestais (SCHUPP, 1993; WUNDERLE-Jr., 1997; GUARIGUATA e PINARD, 1998). Assim, avaliar as variações no espectro de dispersão das comunidades arbóreas é fundamental para a compreensão do funcionamento e dinâmica florestal, e as informações geradas servem de base para o desenvolvimento de estratégias de restauração, manejo e conservação das florestas (WUNDERLE-Jr., 1997; GUARIGUATA e PINARD, 1998; VIEIRA e SCARIOT, 2006).

Estudos em fragmentos de Floresta Atlântica ombrófila do Sudeste vêm indicando que a maioria das espécies arbóreas apresenta síndrome de dispersão zoocórica, cuja importância relativa pode variar de 50\% a 99\% entre as florestas (MORELLATO e LEITÃOFILHO, 1992; TABARELLI et al., 1999; TALORA e MORELLATO, 2000; TABARELLI e PERES, 2002; MEIRA-NETO et al., 2003; CARVALHO et al., 2006). Alguns desses estudos (TABARELLI et al., 1999; TABARELLI e PERES, 2002; CARVALHO et al., 2006) também encontraram variações no espectro de dispersão relacionadas ao estágio sucessional das florestas, e nas florestas preservadas a proporção de espécies zoocóricas supera a das florestas secundárias. Apesar da necessidade de análises em escalas mais amplas para as florestas tropicais, conforme enfatizado por Fuentes (2000), apenas o estudo de Tabarelli e Peres (2002) abordou as variações no espectro de dispersão de espécies arbóreas em escala regional na Floresta Atlântica.

Revista Árvore, Viçosa-MG, v.34, n.6, p.1017-1023, 2010
No intuito de preencher parte dessa lacuna de conhecimento, este trabalho teve como objetivo analisar as diferenças nas proporções das síndromes de dispersão de propágulos de espécies arbóreas entre florestas ombrófilas secundárias e preservadas no Estado do Rio de Janeiro. Partiu-se da hipótese de que as florestas secundárias apresentariam menor riqueza e densidade de espécies arbóreas com síndrome de dispersão biótica (zoocoria), em comparação com as florestas preservadas.

\section{MATERIAL E MÉTODOS}

Foram utilizados os dados de estudos fitossociológicos de 12 fragmentos de Floresta Ombrófila Densa Submontana (50 a 250 m de altitude, sensu VELOSO et al., 1991) localizados no Estado do Rio de Janeiro. Informações sobre os fragmentos florestais encontram-se na Tabela 1. Esses fragmentos foram classificados como floresta secundária (com registro de distúrbio antrópico recente ou há menos de 50 anos) e floresta preservada (sem registros de distúrbio antrópico) de acordo com as descrições nos trabalhos analisados, consultas a antigos moradores do local e conhecimentos do autor. Ao todo foram analisados sete fragmentos florestais secundários e cinco fragmentos florestais maduros.

Foram considerados como indivíduos arbóreos aqueles com DAP $\geq 5,0 \mathrm{~cm}$ (diâmetro à altura do peito =1,30 m acima do nível do solo) (Tabela 1 ). Foram incluídos na análise apenas os indivíduos identificados em nível de espécie e, ou, gênero. A determinação das síndromes de dispersão foi baseada nos trabalhos de Lorenzi (1992, 1998), Barroso et al. (1999) e conhecimentos do autor. Eventualmente, foram consultados especialistas do Jardim Botânico do Rio de Janeiro para espécies em dúvida quanto à identificação.

O número de espécies (riqueza) e o número de indivíduos (densidade) foram extraídos dos trabalhos e utilizados nas análises. As espécies foram classificadas quanto às síndromes de dispersão de suas sementes segundo a terminologia de van der Pijl (1982), em zoocóricas (dispersão por vertebrados), anemocóricas (dispersão por vento) e autocóricas (autodispersão). Posteriormente, as espécies foram classificadas em dois grupos: (1) espécies com síndrome de dispersão biótica (zoocoria) e (2) espécies com dispersão abiótica (anemocoria e autocoria). Como os grupos são complementares, adotou-se a dispersão biótica como critério de comparação. As médias das proporções de 
dispersão biótica entre florestas secundárias e maduras, para riqueza e densidade, foram comparadas pelo teste 'U' de Mann-Whitney (BROWER e ZAR, 1984).

\section{RESULTADOS E DISCUSSÃO}

As espécies com síndrome de dispersão biótica (zoocóricas) prevaleceram nos 12 fragmentos florestais analisados, tanto quando considerada a riqueza quanto a densidade dos indivíduos (Figura 1). A proporção de espécies com síndrome de dispersão biótica variou de $63,7 \%$ a $72,9 \%$ nas florestas secundárias e de $82,3 \%$ a 89,9\% nas florestas preservadas (Figura 1a). Já a densidade de indivíduos com síndrome de dispersão biótica variou de $61,4 \%$ a $72,8 \%$ nas florestas secundárias e de $82,3 \%$ a $90,3 \%$ nas florestas preservadas (Figura 1b).

Esses resultados estão de acordo com os encontrados em outros estudos na Floresta Atlântica ombrófila do Sudeste brasileiro, cuja importância relativa da síndrome de dispersão biótica ultrapassa os 50\% (MORELLATO e LEITÃO-FILHO, 1992; TABARELLI et al., 1999; TALORA e MORELLATO, 2000; TABARELLI e PERES, 2002; MEIRA-NETO et al., 2003; CARVALHO et al., 2006).

Foram encontradas diferenças significativas entre os fragmentos de florestas secundárias e preservadas quando comparadas com a representatividade das síndromes de dispersão biótica, tanto com relação à riqueza de espécies ( $U=35,0 ; P<0,01$ ) quanto com relação à densidade de indivíduos $(U=35,0 ; P<0,01)$. Em ambos os casos, a proporção de espécies com síndrome de dispersão biótica foi maior nas florestas preservadas (Figura 2), suportando a hipótese de que florestas secundárias possuem menores proporções de espécies e indivíduos arbóreos que utilizam a síndrome de dispersão por animais (zoocoria).

Os resultados corroboram os de Tabarelli e Peres (2002) em sua análise das Florestas Ombrófilas Montanas do Sudeste brasileiro, que detectaram aumento gradual na riqueza de espécies zoocóricas paralelamente ao aumento da maturidade das florestas e sugeriram que as mudanças nas estratégias de dispersão durante o processo de regeneração se devem ao balanço entre espécies de fases sucessionais iniciais (pioneiras e secundárias iniciais) e tardias (tolerantes à sombra) que possuem diferentes estratégias de dispersão.

Segundo Tabarelli e Peres (2002), espécies pioneiras e secundárias iniciais são capazes de se desenvolver em condições de alta luminosidade, cujas sementes são normalmente disseminadas por meios abióticos (principalmente vento) ou pela fauna capaz de consumir os pequenos frutos produzidos, especialmente as aves, ao passo que as espécies tardias germinam e se

Tabela 1 - Fragmentos de Floresta Atlântica Ombrófila Densa Submontana (50-250 m) no Estado do Rio de Janeiro, utilizados nas análises da síndrome de dispersão de espécies arbóreas.

Table 1 - Submountaine Ombrophilous Atlantic Forest fragments (50-250 m) at Rio de Janeiro State, Brazil, utilized in the analyses of woody seed dispersal syndrome.

\begin{tabular}{|c|c|c|c|c|c|}
\hline Fragmentos & Município & Coordenadas & $\begin{array}{c}\text { Amostra } \\
\text { (ha) }\end{array}$ & $\begin{array}{c}\text { Inclusão } \\
\text { DAP (cm) }\end{array}$ & Fonte \\
\hline \multicolumn{6}{|l|}{ Florestas secundárias } \\
\hline Região de Imbaú & Silva Jardim & $22^{\circ} 34^{\prime} \mathrm{S}, 42^{\circ} 26^{\prime} \mathrm{O}$ & 1,00 & $\geq 5,0$ & $\overline{\text { Carvalho et al. (2006) }}$ \\
\hline REBIO Poço das Antas (1) & Silva Jardim & $22^{\circ} 30^{\prime} \mathrm{S}, 42^{\circ} 15^{\prime} \mathrm{O}$ & 0,25 & $\geq 10,0$ & Neves (1999) \\
\hline REBIO Poço das Antas (2) & Silva Jardim & $22^{\circ} 30^{\prime} \mathrm{S}, 42^{\circ} 15^{\prime} \mathrm{O}$ & 0,25 & $\geq 10,0$ & Neves (1999) \\
\hline REBIO Poço das Antas (3) & Silva Jardim & $22^{\circ} 30^{\prime} \mathrm{S}, 42^{\circ} 15^{\prime} \mathrm{O}$ & 0,22 & $\geq 5,0$ & Pessoa (2003) \\
\hline REBIO Poço das Antas (4) & Silva Jardim & $22^{\circ} 30^{\prime} \mathrm{S}, 42^{\circ} 15^{\prime} \mathrm{O}$ & 0,57 & $\geq 5,0$ & Pessoa (2003) \\
\hline REBIO Poço das Antas (5) & Silva Jardim & $22^{\circ} 30^{\prime} \mathrm{S}, 42^{\circ} 15^{\prime} \mathrm{O}$ & 0,65 & $\geq 5,0$ & Pessoa (2003) \\
\hline Fazenda Rio Vermelho & Rio Bonito & $22^{\circ} 44^{\prime} \mathrm{S}, 42^{\circ} 33^{\prime} \mathrm{O}$ & 0,40 & $\geq 5,0$ & Carvalho et al. (2007) \\
\hline \multicolumn{6}{|l|}{ Florestas preservadas } \\
\hline REBIO União & Rio das Ostras & $22^{\circ} 25^{\prime} S, 42^{\circ} 02^{\prime} \mathrm{O}$ & 0,40 & $\geq 10,0$ & Rodrigues (2004) \\
\hline E.E.E. Paraíso (1) & Cachoeiras de Macacú & $22^{\circ} 26^{\prime} \mathrm{S}, 42^{\circ} 50^{\prime} \mathrm{O}$ & 1,00 & $\geq 10,0$ & Guedes-Bruni (1998) \\
\hline E.E.E. Paraíso (2) & Cachoeiras de Macacú & $22^{\circ} 26^{\prime} \mathrm{S}, 42^{\circ} 50^{\prime} \mathrm{O}$ & $0,56 *$ & $\geq 5,0$ & Kurtz \& Araujo (2000) \\
\hline Região do Imbé (1) & Campos dos Goytacazes & $21^{\circ} 48^{\prime} \mathrm{S}, 14^{\circ} 40^{\prime} \mathrm{O}$ & 0,60 & $\geq 10,0$ & Moreno et al. (2003) \\
\hline Região do Imbé (2) & Campos dos Goytacazes & $21^{\circ} 48^{\prime} \mathrm{S}, 14^{\circ} 40^{\prime} \mathrm{O}$ & 0,60 & $\geq 10,0$ & Moreno et al. (2003) \\
\hline
\end{tabular}

* Método de ponto-quadrante; REBIO - Reserva Biológica Federal; e E.E.E. - Estação Ecológica Estadual.

* Quarter-point method; REBIO - Federal Biological Reserve; and EEA - State Ecological Station. 
$\square$ Biótica $\square$ Abiótica


Figura 1 - Distribuição do (a) número de espécies e (b) número de indivíduos arbóreos com síndromes de dispersão biótica e abiótica nas florestas secundárias e preservadas.

Figure 1 -Distribution of the (a) number of species, and (b) number of woody individuals with biotic and abiotic dispersal modes in the secondary and mature forests.
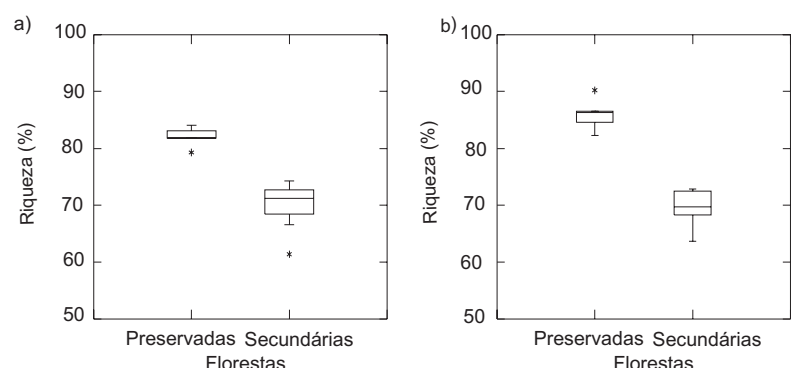

Figura 2 - Comparação entre (a) número de espécies e (b) número de indivíduos arbóreos com dispersão biótica nas florestas secundárias $(n=7)$ e preservadas $(n=5)$. Mediana, desvio-padrão e valores máximo e mínimo são apresentados.

Figure 2 - Comparison of the (a) number of species and (b) number of woody individuals with biotic dispersal mode in the secondary $(n=7)$ and mature $(n=5)$ forests. Median, standard deviation, and maximum and minimum values were presented. desenvolvem em condições de sombreamento do dossel e possuem sementes maiores, disseminadas seja por gravidade, seja, principalmente, por grandes animais, especialmente mamíferos.

Estudos nas florestas secundárias aqui analisadas já haviam indicado predominância na riqueza e densidade de espécies de fases sucessionais iniciais (secundárias iniciais e pioneiras) sobre as tardias (secundárias tardias). Nessas florestas, as espécies pioneiras e secundárias iniciais estão representadas, principalmente, pelas famílias Euphorbiaceae, Leguminosae, Meliaceae e Sapindaceae (NEVES, 1999; PESSOA, 2003; CARVALHO et al., 2006; CARVALHOetal., 2007), cujos frutos sãopredominantemente anemocóricos (Leguminosae) ou zoocóricos (Euphorbiaceae, Meliaceae e Sapindaceae) consumidos e disseminados pela avifauna (CORREIA, 1997).

Paralelamente ao sucesso da disseminação de espécies arbóreas de fases iniciais (principalmente aquelas pertencentes às famílias Euphorbiaceae, Meliaceae e Sapindaceae), todas as florestas secundárias apresentaram decréscimo na densidade das espécies pertencentes às famílias Lauraceae, Myrtaceae e Sapotaceae (Figura 3). Na Floresta Atlântica do Sudeste brasileiro, essas espécies são, em sua maioria, de fases sucessionais tardias (secundárias tardias) (TABARELLI et al., 1999; TABARELLI e PERES, 2002; CARVALHO et al., 2006), cujos frutos são consumidos e suas sementes disseminadas, em sua maioria, por animais frugívoros de médio a grande porte, como algumas espécies de aves e mamíferos, principalmente primatas (TABARELLI et al., 1999; TABARELLI e PERES, 2002). Há de se considerar que essas famílias tendem a sofrer maior pressão pelo seu valor comercial (qualidade da madeira) (CARVALHO 2005; VILLELA et al., 2006), e sua redução nos fragmentos pode ser decorrência tanto dos efeitos sinérgicos da fragmentação florestal pretérita (aumento na pressão de extração seletiva, redução de área, efeito de borda, mudanças microclimáticas, aumento de suscetibilidade a danos por insetos e patógenos) (TABARELLI et al., 2004; CARVALHO 2005; MELO et al., 2007), quanto pela diminuição ou ausência da fauna dispersora associada (WUNDERLE-Jr., 1997).

Assim como observado na maioria das regiões neotropicais, o papel dos grandes dispersores na Floresta Atlântica esbarra no fenômeno denominado "floresta vazia”, onde árvores maduras são privadas das interações essenciais com seus dispersores como resultado da sua 


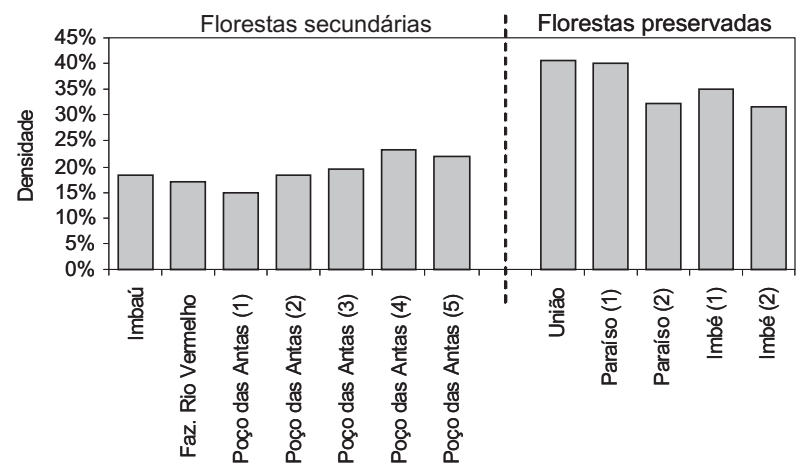

Figura 3 - Comparação da densidade das espécies arbóreas pertencentes às famílias Lauraceae, Myrtaceae e Sapotaceae entre as florestas secundárias e preservadas.

Figure 3-Comparison of the density of woody species belonging to the families Lauraceae, Myrtaceae and Sapotaceae in the secondary and mature forests.

eliminação pela caça e fragmentação florestal (REDFORD, 1992). Nesse caso, como a composição da fauna dispersora influencia fortemente no recrutamento de plântulas no processo de sucessão florestal, sua redução e, ou, alteração representa enorme risco tanto para a regeneração florestal quanto para a colonização de novas áreas secundárias (SCHUPP, 1993; WUNDERLE-Jr., 1997; GUARIGUATA e PINARD, 1998).

Considerando o alto grau de fragmentação Florestal das Florestas Ombrófilas Submontanas do Estado do Rio de Janeiro e o longo período em que seus remanescentes se encontram vulneráveis a ações antrópicas (Fundação SOS MATAATLÂNTICA, 2002), o decréscimo na densidade de espécies arbóreas dispersas por grandes vertebrados frugívoros aparenta ser um dos reflexos da fragmentação pretérita associado à diminuição ou ausência das populações dos grandes dispersores nessas florestas, conforme já enfatizado por Tabarelli e Peres (2002) e Tabarelli et al. (2004). Por esse caminho, mesmo essas áreas em regeneração (florestas secundárias) podem-se tornar incapazes de retornar à sua condição mais próxima possível da original sem a presença da fauna dispersora adequada(SCHUPP, 1993; WUNDERLE-Jr, 1997; GUARIGUATA e PINARD, 1998).

\section{CONCLUSÕES}

Conforme a hipótese levantada, as florestas secundárias apresentaram menor riqueza e densidade de espécies com dispersão biótica em relação às preservadas, além de menor densidade de espécies zoocóricas pertencentes a famílias dispersadas por grandes vertebrados frugívoros (Lauraceae, Myrtaceae e Sapotaceae). Segundo estudo do Ministério do Meio Ambiente (2000), as florestas analisadas são consideradas de relevante interesse conservacionista, pois ainda detêm importante fração da fauna e flora local e de grande representatividade em nível regional, incluindo algumas espécies ameaçadas ou vulneráveis à extinção, como o primata mico-leão-dourado (Leontopithecus rosalia). Com base nos resultados, sugere-se que os programas de recomposição e manejo florestal nessa região considerem as interações entre plantas e seus dispersores como uma das principais diretrizes, com enfoque especial nas aves e nos grandes vertebrados frugívoros, como os primatas, pois, mesmo protegidas essas florestas secundárias, podem ter problemas de regeneração e manutenção das populações arbóreas em longo prazo sem a presença da fauna dispersora adequada.

\section{AGRADECIMENTOS}

Aos pesquisadores Jeanine M. Felfili, José R.R. Pinto, Daniel L.M. Vieira, Benedito A.S. Pereira e Felipe N.A.A. Rego, pela revisão do manuscrito; aos pesquisadores do Jardim Botânico do Rio de Janeiro, em especial a João Marcelo A. Braga, pelo auxílio na determinação das síndromes de dispersão; a Felipe N.A.A. Rego, pelo auxílio na análise dos dados; e aos Departamentos de Ecologia e de Engenharia Florestal da Universidade de Brasília (UnB) e ao Laboratório de Ciências Ambientais da Universidade Estadual do Norte Fluminense (UENF), pelo apoio logístico.

\section{REFERÊNCIAS}

BARRoso, G. M. et al. Frutos e sementes: morfologia aplicada à sistemática de Dicotiledôneas. Viçosa, MG: Universidade Federal de Viçosa, 1999. 444p.

BRASIL. Ministério do Meio Ambiente.

Avaliação e ações prioritárias para a conservação da biodiversidade da Mata Atlântica e Campos Sulinos. Brasília: 2000.

BROWER, J. E.; ZAR, J. H. Field and laboratory methods for general ecology. 2.ed. Ames: W.C. Brown Company Publishers, 1984. 255p.

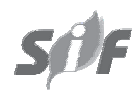

Revista Árvore, Viçosa-MG, v.34, n.6, p.1017-1023, 2010 
BROWN, S.; LUGO, A. E. Tropical secondary forests. Journal of Tropical Ecology, v.6, n.1, p.1-32, 1990.

CARVALHO, F. A. Efeitos da fragmentação florestal na florística e estrutura de fragmentos de Mata Atlântica submontana na região de Imbaú, município de Silva Jardim, RJ. 2005. 124f. Dissertação (Mestrado em Biociências e Biotecnologia) - Universidade Estadual do Norte Fluminense, Campos dos Goytacazes, 2005.

CARVALHO, F. A.; NASCIMENTO, M. T.; BRAGA, J. M. A. Composição e riqueza florística do componente arbóreo da Floresta Atlântica submontana na região de Imbaú, município de Silva Jardim, RJ. Acta Botanica Brasílica, v.20, n.3, p.727-740, 2006.

CARVALHO, F. A.; NASCIMENTO, M. T.; BRAGA, J. M. A. Estrutura e composição florística do estrato arbóreo de um remanescente de Mata Atlântica submontana no município de Rio Bonito, RJ, Brasil (Mata Rio Vermelho). Revista Árvore, v.31, n.4, p.717-730, 2007.

CHAZDON, R. L. et al. Rates of change in tree communities of secondary Neotropical forests following major disturbances. Philosophical Transactions of the Royal Society B, v.362, p.273-289, 2007.

CORREIA, J. M. S. Utilização de espécies frutíferas da Mata Atlântica na alimentação da avifauna da Reserva Biológica de Poço das Antas. 1997. $79 f$. Dissertação (Mestrado em Ecologia) -

Universidade de Brasília, Brasília, 1997.

FUENTES, M. Frugivory, seed dispersal and plant community ecology. Trends in Ecology and Evolution, v.15, n.12, p.487-488, 2000.

FUNDAÇÃO SOS MATA ATLÂNTICA. Atlas da evolução dos remanescentes florestais e ecossistemas associados no domínio da Mata Atlântica no período 1995-2000. São Paulo: SOS Mata Atlântica/INPE/ISA, 2002.

GENTRY, A. H. Patterns of neotropical plant species diversity. Evolutionary Biology, v.15, n.1, p.1-84, 1982.

Revista Árvore, Viçosa-MG, v.34, n.6, p.1017-1023, 2010
GUARIGUATA, M. R.; PINARD, M. A. Ecological knowledge of regeneration from seed in neotropical forest trees: Implications for natural forest management. Forest Ecology and Management, v.112, n.1, p.87-99, 1998.

GUEDES-BRUNI, R. R. Composição, estrutura e similaridade florística de dossel em seis unidades de Mata Atlântica no Rio de Janeiro. 1998. $231 \mathrm{f}$. Tese (Doutorado em Ecologia) - Universidade de São Paulo, São Paulo, 1998.

HOWE, H. F.; SMALLWOOD, J. Ecology of seed dispersal. Annual Review of Ecology and Systematics, v.13, p.201-228, 1982.

KURTZ, B. C.; ARAUJO, D. S. D. Composição florística e estrutura do componente arbóreo de um trecho de Mata Atlântica na Estação Ecológica Estadual do Paraíso, Cachoeira de Macacu, Rio de Janeiro, Brasil. Rodriguésia, v.51, p.69-112, 2000.

LORENZI, H. Árvores Brasileiras: manual de identificação e cultivo de plantas arbóreas nativas do Brasil. Nova Odessa: Plantarum, 1992. v.1.352p.

LORENZI, H. Árvores Brasileiras: manual de identificação e cultivo de plantas arbóreas nativas do Brasil. Nova Odessa: Plantarum, 1998. v.2. 368p.

MEIRA-NETO, J. A. et al. Origem, sucessão e estrutura de uma floresta de galeria periodicamente alagada em Viçosa-MG. Revista Árvore, v.27, n.4, p.561-574, 2003.

MELO, F. P. L.; LEMIRE, D.; TABARELLI, M. Extirpation of large-seeded seedlings from the edge of a large Brazilian Atlantic forest fragment. Ecoscience, v.14, n.1, p.124-129, 2007.

MORELLATO, L. P. C.; LEITÃO-FILHO, H. F. Padrões de frutificação e dispersão na serra do Japi. In: MORELLATO, L. P. C. História natural da serra do Japi: ecologia e preservação de uma área florestal no sudeste do Brasil. Campinas: Universidade de Campinas, 1992. p.112-140.

MORENO, M. R.; NASCIMENTO, M. T.; KURTZ, B. C. Estrutura e composição florística do estrato arbóreo em duas zonas altitudinais na Mata Atlântica de encosta da região do Imbé, RJ. Acta Botanica Brasilica, v.17, n.3, p.371-386, 2003. 
NEVES, G. M. S. Florística e estrutura da comunidade arbustivo-arbórea em dois remanescentes de Floresta Atlântica secundária - Reserva Biológica de Poço das Antas, Silva Jardim, RJ. 1999. 115f. Dissertação (Mestrado em Ciências Biológicas) - Universidade Federal do Rio de Janeiro, Rio de Janeiro, 1999.

PESSOA, S. V. A. Aspectos da

fragmentação em remanescentes

florestais da planície costeira do estado do Rio de Janeiro. 2003. 112 f. Dissertação (Mestrado em Ciências Florestais) Universidade Federal Rural do Rio de Janeiro, Seropédica, 2003.

REDFORD, K. H. The empty Forest. Bioscience, v.42, n.6, p.412-422, 1992.

RODRIGUES, P. J. F. P. A vegetação da Reserva Biológica União e os efeitos de borda na Mata Atlântica

fragmentada. 2004. 136f. Tese (Doutorado em Biociências e Biotecnologia) - Universidade Estadual do Norte Fluminense, Campos dos Goytacazes, 2004.

SCHUPP, E. W. Quantity, quality and the effectiveness of seed dispersal by animals. Vegetatio, v.107/108, p.15-29, 1993.

TABARELLI, M.; MANTOVANI, W.; PERES, C. A. Effects of habitat fragmentation on plant guild structure in the montane Atlantic forest of Southeastern Brazil. Biological

Conservation, v.91, 2/3, p.119-127, 1999.
TABARELLI, M.; PERES, C. A. Abiotic and vertebrate seed dispersal in Brazilian Atlantic Forest: implications for forest regeneration. Biological Conservation, v.106, n.2, p.165-176, 2002.

TABARELLI, M.; SILVA, J. M. C.; GASCON, C. Forest fragmentation, synergisms and the impoverishment of neotropical forests.

Biodiversity and Conservation, v.13, n.7, p.1419-1425, 2004.

TALORA, D. C.; MORELLATO, P. C. Fenologia de espécies arbóreas em floresta de planície litorânea do sudeste do Brasil. Revista Brasileira de Botânica, v.23, n.1, p.13-26, 2000.

van der PIJL, L. Principles of dispersal in higher plants. Berlim: Springer-Verlag, 1982. 634p.

VELOSO, H. P., RANGEL FILHO, A. L. R.; LIMA, J. C. A. Classificação da vegetação brasileira adaptada a um sistema universal. Rio de Janeiro: IBGE, 1991. 123p.

VIEIRA, D. L. M.; SCARIOT, A. Principles of natural regeneration of tropical dry forests for restoration. Restoration Ecology, v.14, n.1, p.11-20, 2006.

VILLELA, D. M. et al. Effect of selective logging on forest structure and nutrient cycling in a seasonally dry Brazilian Atlantic forest. Journal of Biogeography, v.33, n.3, p.506-516, 2006.

WUNDERLE Jr., J. M. The role of animal seed dispersal in accelerating native forest regeneration on degraded tropical lands. Forest Ecology and Management, v.99, n.1/2, p.223-235, 1997. 